\title{
VACUUM ELECTRONICS
}

\section{APPLICATION OF FOCUSED CHARGE- PARTICLE BEAMS OF IN MANUFACTURING OF NANOCOMPONENTS}

\author{
G.S. Vorobyov", A.G. Ponomarev², A.A. Ponomareva 1 , \\ A.A. Drozdenko ${ }^{1}, \mathcal{E}$ A.A. Rybalko ${ }^{1}$ \\ 1) Sumy State University, 2 Rymski-Korsakov St., Sumy, 40007, Ukraine \\ 2) Institute of Applied Physics, 58, Petropavlovskaya St., Sumy, 40030, Ukraine \\ ^Address all correspondence to G.S. Vorobyov E-mail: vp@sumdu.edu.ua \\ Application of focused beams of medium energy light ions, electrons and low energy heavy ions is \\ considered for the technology of manufacturing of small-dimension components. Physical principles \\ applied as the basis for interaction of the above beams with resistive materials are described. The \\ proton beam lithography is considered as a new technology possessing high potential capabilities for \\ various applications like micro-optics and nanoelectronics of terahertz wave band.
}

KEY WORDS: manufacturing, focused beam, nanoelectronics, beam litography

\section{INTRODUCTION}

The problem of development of new tools and technologies for manufacturing of nanomaterials and nano-objects still remains actual nowadays. Among the wide scope of physical principles on the basis of which new tools and technologies could be developed, the special attention is paid to the focused beams of charged particles with the low and medium energies. Their dimensions amount to several nanometers. Focused beams of electrons are widely applied in electron beam lithography (EBL, e-beam lithography) [1,2]. Focused beams of heavy ions are applied presently for creation of 3D nanodimensional components in FIB (focusing ion beam) devices [3,4] due to their capability to sputter the atoms of the processed sample. The beams of $\mathrm{MeV}$-energy light ions were initially applied for the purpose of local element analysis of the substance in nuclear scanning microprobes. During recently application of nuclear microprobes has made a great progress in the ion beam lithography in order to develop 3D nanodimensional components $[5,6]$ as well. This technology got the name of 'p-beam writing' (PBW). This brief review pays special attention to considering the state-of-the-art of this technology and its further development. 


\section{PARTICULARITIES OF CHARGED PARTICLE PROPAGATION IN THE SUBSTANCE}

Depending upon their energy the various types of charged particles are differently interacting with the substance in the solid state phase. Scattering upon the atom electrons is the basic mechanism of interaction between the low-energy electrons from the focused beam and the substance. As the result of that primary electrons are multiply deviating by a large angle thus forming up a pear-like shape of the ionization domain appearing in the touch point of the primary focused beam. This electron beam particularity is hampering creation of 3D structures possessing high-value aspect ratio. Another negative factor influencing upon the substance irradiation process is also represented by the proximity effect that requires performing additional correction while determining the proper dose.

Scattering of incident ions upon the nuclei of target atoms is a typical mechanism of interaction with the substance for the focused beams of low-energy heavy ions. Due to the pulse transfer from the beam ions to atoms of the near-surface layer of the material there occurs their rearrangement that results in chemical and structural transformations like sputtering of atomic and molecular formations.

Focused beams of light ions with the energy of several $\mathrm{MeV}$ can interact both with electrons and with the nuclei of the atoms of irradiated material. Owing to a large difference in mass values ion-to-electron interactions cannot substantially vary the incident ion propagation trajectory, which is little different from the straight line. Considering that the value of the energy lost by the electron in such interactions is small multiple cases of interaction between the electrons and the atoms of the material might occur up to the time when the ion loses its kinetic energy completely. A distinctive feature of the beams of medium energy light ions as compared to the electron beams is in the fact that there are practically absent secondary electrons with the energy capable of exerting a substantial influence upon the material irradiation dose. The ion penetration depth for the selected material depends upon its energy and is rigidly determined that is an important factor while creating multilevel 3D objects within single-layer resistive materials.

\section{PROBE SYSTEMS FOR FORMATION OF THE CHARGE- PARTICLE BEAMS}

The principles of electron beam formation in EBL systems are similar to those of the scanning electron microscope (SEM) where the electron beam is focused into a spot upon the sample with the help of electromagnetic lenses with axial symmetry and scanned in the required domain in order to obtain an image. Basic differences between EBL and SEM are in the fact that within the EBL systems the following elements are very important: high-precision positioning of the sample; system for measuring of the spot dimensions; beam current measurement for normalizing of the dose; high-speed system for dropping of the beam while shifting the sample. 
General block diagram of forming of the focused beam of low energy heavy ions in FIB devices possesses the principles of focused beam formation similar to those applied in the system of electron beams focusing, the main difference in this case is application of the beam of gallium ions $\left(\mathrm{Ga}^{+}\right)$instead of the electron beam [7].

Like in the case with the devices applied in the e-beam lithography the specialized scanning microprobe serving the purposes of the ion beam lithography consists of the ion gun, probe-forming system and the target chamber (Fig. 1). Here the electrostatic accelerator with the analyzing magnet acts as an ion gun. At the accelerator output it is created a beam of accelerated light ions $\left(\mathrm{H}^{+}, \mathrm{H}_{2}^{+}, \mathrm{He}^{+}\right)$with the energy of up to several MeV. Subsequently its formation is occurred with the help of the object and angular collimators in order to attain that the focusing system consisting of the multiplet of magnetic quadruple lenses would provide for focusing of the beam upon the target into a spot with the minimally possible dimensions. Currently, the best results have been attained on the device in the Center for Ion Beam Applications (CIBA) at Singapore National University [8]. Upon that microprobe device there are obtained the spot dimensions upon the target of $290 \times 450 \mathrm{~nm}^{2}$ with the proton beam of $50 \mathrm{pA}$ and the $\mathrm{H}_{2}^{+}$ion beam dimensions of $35 \times 75 \mathrm{~nm}^{2}$ with the current at the level of $1 \mathrm{pA}$.

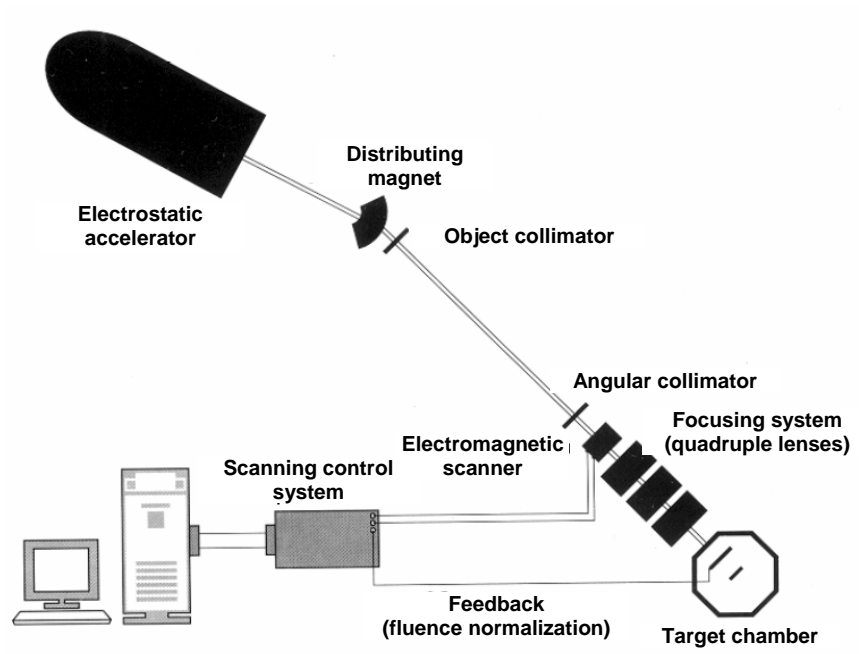

FIG. 1: Block diagram of the focused beam formation of medium energy light ions within the nuclear scanning microprobe for the purposes of the p-beam writing technology

Despite the significant progress achieved in parameters of nuclear microprobe devices and a number of preferential properties of focused beams of $\mathrm{MeV}$ energy ions as compared to similar electron beams and the beams of low energy heavy ions, the perspectives of manufacturing of industrial samples are related primarily to development of new layouts for arrangement of the microprobes allowing to create a more compact device. The papers [9-11] suggest one of the options of such an 
arrangement permitting to substantially decrease the device dimensions and to improve beam parameters upon the target.

\section{TECHNOLOGICAL PROCESSES FOR DEVELOPMENT OF NANOCOMPONENTS WITH APPLICATION OF FOCUSED BEAMS OF CHARGED PARTICLES}

While propagating within the sample the charged particles cause variation of physical and chemical properties of the irradiated domain. While processing the sample with the relevant developer and using electrochemical etching in some cases, the irradiated domain is deleted. In this case the resistive material is called positive and vice versa deleting of the non-irradiated domain is inherent to negative resistive materials. Figure 2 demonstrates the 3D nanodimensional components obtained with the help of the PBW technology in the PMMA layer with the thickness of $350 \mathrm{~nm}$ (a) and in the SU-8 single-layer material (b).

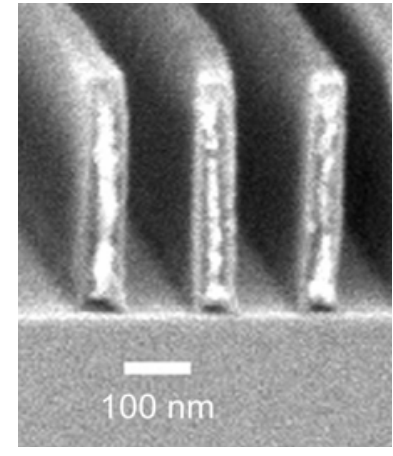

a)

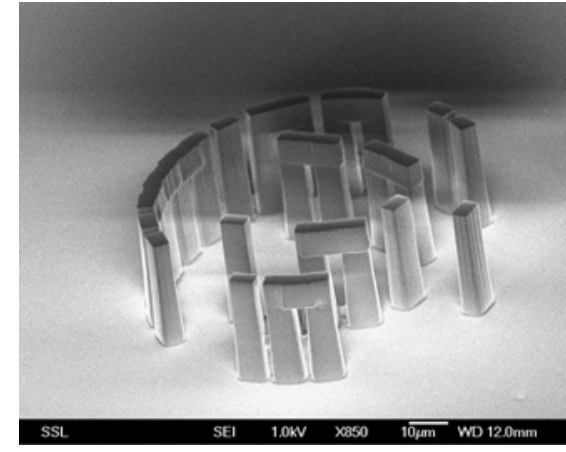

b)

FIG. 2: Images of the 3D nanodimensional components obtained with the help of the p-beam writing technology [5]

The basic distinctive feature of application of the technology using the proton beam as compared to the electron beam is application of resistive materials having a less thickness when the component dimensions are decreasing. This is a consequence of electrons scattering at their propagation in the sample; in so doing the components are practically two-dimensional. At the same time the proton beam is practically not expanded within the resistive materials, that allows creating 3D nanocomponents in this case.

The silicon, on the basis of which various element of microwave devices could be realized by means of using different technologies, is one of the most promising materials in nano-electronics. Provided in the paper [12] results of research and development of technological processes of deep anisotropic etching of 
micromechanical elements for microwave devices prove that it is impossible to obtain separate elements of the preset shape using liquid etching of silicon only. For this reason the technologies of using dry etching with applying plasma sources appear to have considerable promise. The basic of them are - reactive ion etching, reactive ion etching with concentration by the magnetic field, electron cyclotrone resonance, induced coupled plasma (ICP) etc. To provide for deep anisotropic etching of the silicon using the ICP source there are applied nowadays a number of devices having different shapes, which are distinguished by the level of their technological parameters. In particular, Fig. 3 shows the image of an etched surface profile of the standard silicon plate (100) with the diameter of $100 \mathrm{~mm}$ for the etching depth of about $100 \mu \mathrm{m}$.
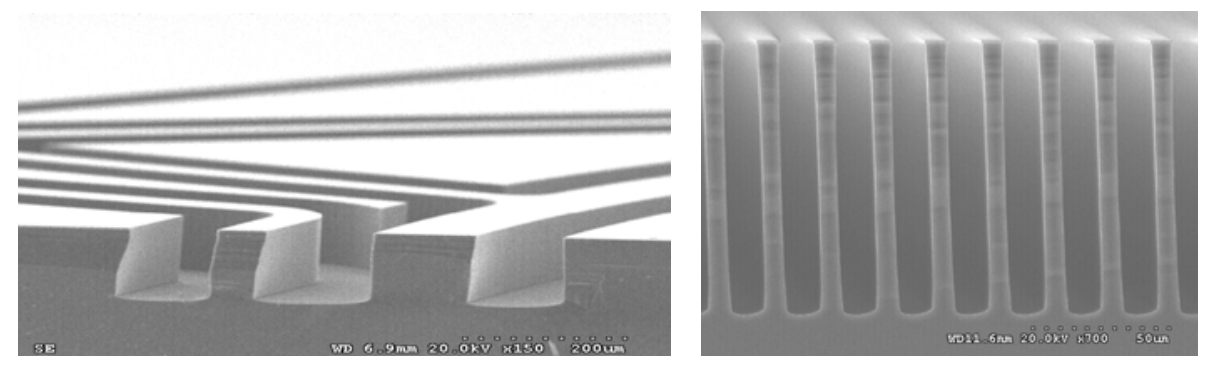

FIG. 3: Chipping of silicon plates after performing of the etching procedure [12]

It is perspective to create structures based on the substrate of the silicon with its oxide containing ion tracks, within which by means of the method of subpotential electrochemical depositing there are formed homogeneous nanocompositions or multilayer nanostructures with alternating layers consisting of ferromagnetic and nonmagnetic nanoparticles [13]. Investigation of such structures creates preconditions for modeling of electron devices with the Schottky barrier possessing non-linear voltampere characteristics including the negative differential impedance. Realization of the formulated idea would allow developing magnetic field sensors with enhanced sensitivity [14].

As an example of realization of the above technology Fig. 4 a provides the isometric image of the $\mathrm{SiO}_{2}$ surface with etched ion tracks filled with $\mathrm{Cu}$. The image is taken from the paper [13].

From the above consideration of the technologies based on the direct methods of dry ion etching of the silicon, it is evident that typical dimensions of the components are of the value of the order of a micrometer. Changing to technological processes with application of the PBW technologies would allow decreasing typical dimensions of the components by more than one order. This technology permits creation of 3D nanocomponents within the mass volume of the silicon with high aspect ratio (Fig. 4(b)). Similar mechanism of creation of 3D structures is also applied for GaAs [15]. 


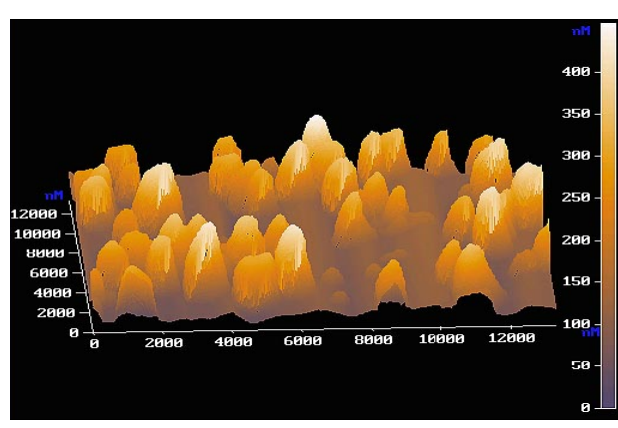

a)

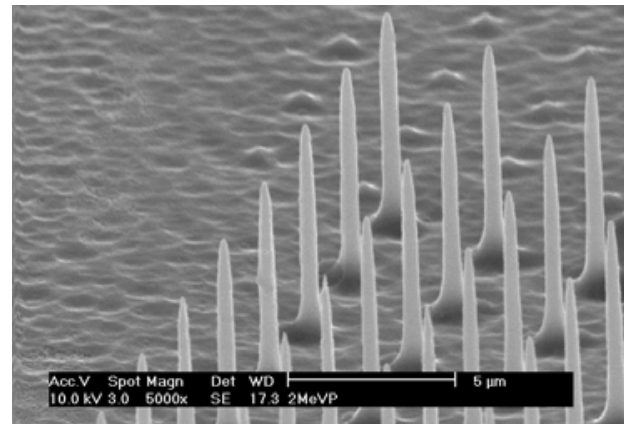

b)

FIG. 4: Nanostructures obtained in the silicon with application of various technologies: $\mathrm{a}-[13], \mathrm{b}-[5]$

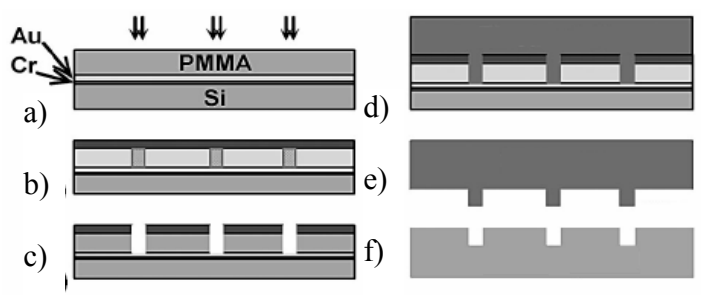

FIG. 5: Diagram representation of the process of die manufacturing using the PBW technology [15]

The processes using light projection, X-ray or electron and ion lithography are the most suitable for mass manufacturing of small-dimension structures positioned upon a large area and with high density. Nevertheless, increasing technological hardships and expected substantial increase of costs of production for the components with typical dimensions less than $100 \mathrm{~nm}$ are predetermining the search for alternative solutions. For this reason the methods of lithography using focused beams of charged particles in combination with nano die forming might appear more preferable in several cases [16]. As an example, Fig. 5 demonstrates the sequence of production process for manufacturing of 3D Ni dies using PBW technologies and galvanic coating with the nickel. Here, the silicon substrate $\operatorname{Si}(100)$ is initially coated by layers of $\operatorname{Cr}(20 \mu \mathrm{m})$ and $\mathrm{Au}(200 \mu \mathrm{m})$ for better adhesion and electric conductivity and then with the help of the centrifuge it is applied a layer of the RMMA resistive material, which is subject to irradiation with a focused beam of protons having the energy of $2 \mathrm{MeV}$ (Fig. 5(a)). Later on the second metallized layer is applied to the upper surface. It acts as the die base and provides for the conditions of conductivity at the galvanic coating (Fig. 5(b)). As the result of processing by the developing agent it is formed a $3 \mathrm{D}$ structure (Fig. 5(c)), upon which the nickel coating is applied with the help of the galvanic process (Fig. 5(d)). 
Finally it is performed separation of the die from the template (Fig. 5(e)) and nano die forming (Fig. 5(f)).

\section{SCOPE OF APPLICATION}

\subsection{Microphotonics and microoptics}

Micro-optics and microphotonics occupy a special place among the multiplicity of applications of micro- and nanocomponents that may be created using the PBW technology. There are two means, with the help of which the PBW technology can be used for applications in microoptics and microphotonics. The first one includes direct forming of small-dimension components of polymers coated with the help of a centrifuge upon the relevant substrate like glass or thermally oxidized silicon plate. In this case, the substrate and the coated material must possess lower refraction coefficient than the material of the fiber optic core. Another technology of forming of waveguides in the mass volume of the polymer or quartz glass using PBW includes direct ion beam modification without any developing step [18]. This is attained due to application of processes occurring at the final stage of ion propagation in the sample, when the latent waveguide channel is created within the substrate.

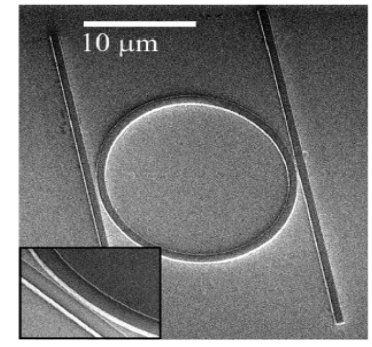

a)

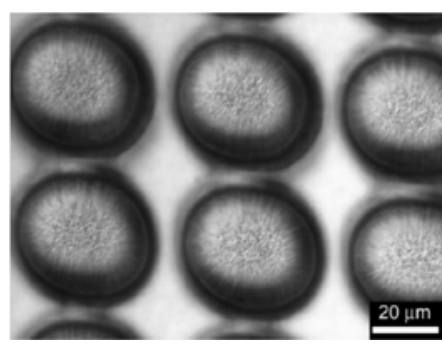

b)

FIG. 6: Microoptical elements created with application of the PBW technology

As an example, Fig. 6 shows SEM images of the ring resonator manufactured within a layer of the SU-8 resistive material upon the silicon substrate (Fig. 6(a), [18]) and the microlens array performed of the RMMA resistive material having the thickness of $15 \mu \mathrm{m}$ (Fig. 6(b), [8]).

\subsection{Nano-electronics of terahertz band}

Development of high-resolution sensitive elements for terahertz frequency bandwidth is an actual problem due to a number of existing international projects of radio astronomy as well as the projects with the objective of studying the Earth atmosphere. 
The basic issues of arrangement of receivers in the given bandwidth are solved by application of solid-state heterodyne oscillation sources and the mixers based on the effect of electron heating in the superconductor (Hot Electron Bolometer) because such mixers have no competition analogs in this bandwidth. By now these mixers have been successfully realized at the frequencies of the order of $3 \mathrm{GHz}$. However, several research projects are related to development of the mixers optimized for higher frequencies; e.g. within the framework of the SOFIA project [19] it is developed a heterodyne receiver for $4.8 \mathrm{THz}$. Principal opportunity for creation of the mixer for the given bandwidth is shown in the paper [20] where the method of electron and photo lithography is applied for its realization. As an example, Fig. 7 provides photos of the central part of the mixer obtained with the scanning electron microscope.
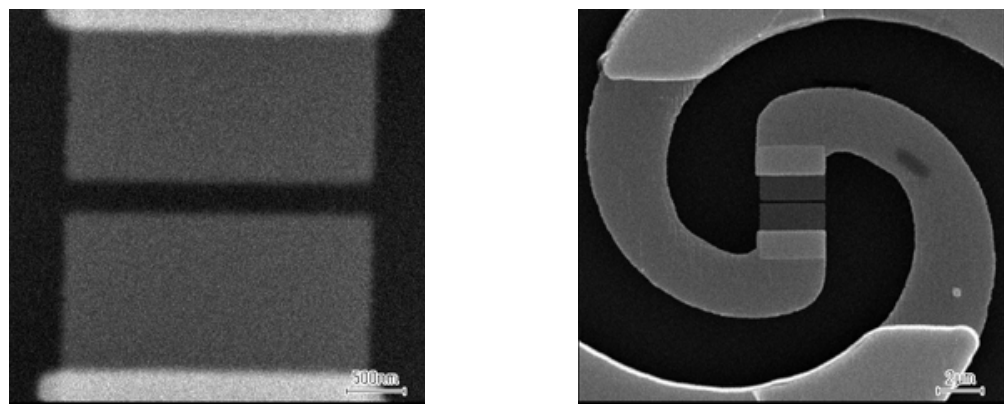

FIG. 7: Image of the helix (left) and of the central part of the helix with the $\mathrm{NbN}$ bridge (right) obtained with the scanning electron microscope [20]

The mixers were made of NbN films with the thickness of 2 to $3.5 \mathrm{~nm}$ upon a silicon substrate with a $\mathrm{MgO}$ buffer sublayer and possessed the temperature of superconducting transition of 9 to $11 \mathrm{~K}$. The best value of the noise temperature of the receiver on the basis of the electron heated mixer amounted to $1300 \mathrm{~K}$ and $3100 \mathrm{~K}$ at the heterodyne frequencies of 2.5 and $3.8 \mathrm{THz}$ correspondingly.

In the radio astronomy devices and tools the preference is given to solid state heterodyne sources within the terahertz band due to their small dimensions, low weight and power consumption requirements, despite low output power level, which is not exceeding $1 \mu \mathrm{W}$ at the frequency of $2 \mathrm{THz}$ that to a significant extent complicates the problem of development of mixers for a low level of output power. Thus, there still remains actual the problem of realization of low-voltage electrovacuum oscillation sources within the terahertz wavelength band possessing higher values of power levels as compared to those of solid state oscillators.

By the present time this problem can be solved by means of using in the vacuum electronics of planar periodic metal dielectric structures (MDS) [21-24]. Block diagrams of such devices that could be realized on the basis of the above described technologies and new types of dielectrics with larger values of dielectric permeability and low loss values at high frequencies [25], are provided in Fig. 8. 


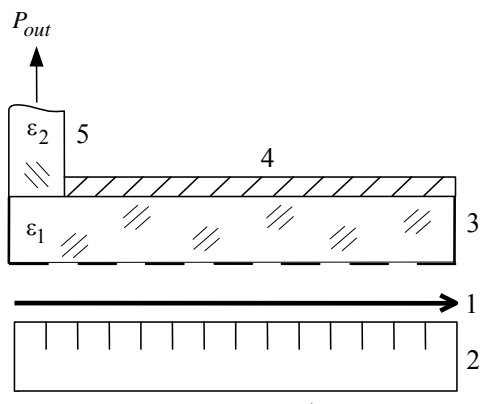

a)

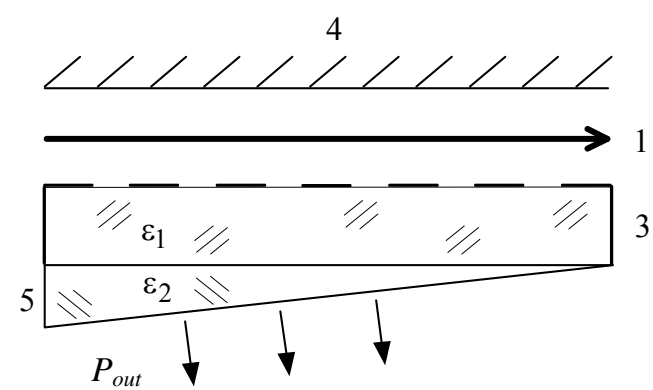

b)

FIG. 8: Block diagrams of terahertz frequency band electrovacuum oscillation sources: 1 - electron beam; 2 - periodic structure; 3 - MDS with diffraction strip-like grating; 4 - screen (mirror); 5 - energy output device

Block diagram of the oscillator in Fig. 8(a) suggests modulation of the electron beam upon a backward wave of the periodic structure (position 2) with subsequent excitation of the Cherenkov oscillation harmonic in MDS (position 3). Within the oscillator in Fig. 8(b) it is applied the mode of abnormal diffraction oscillation [23], which is realized at substantially less values of accelerating voltages of the electron flux compared to those of the Cherenkov oscillation harmonic.

At practical realization of the above block diagrams of sources of oscillation it is necessary to solve a set of problems related to the technology of manufacturing of main units of the device - the electrodynamic system, the low-voltage source of electrons and the focusing magnetic system. The above considered technological processes - like, for instance, nano die forming - eliminate all problems related to manufacturing of both reflecting and ribbon (applied upon the dielectric layer) diffraction gratings with the micron period.

The estimates provided in [21] demonstrate that within the submillimeter and infrared wavelength bands the thickness of the electron beam has to be decreased up to the values $0.04 \mathrm{~mm}$. This problem can be solved nowadays due to application of the matrix-type field [26] and slot L-cathodes [27], the fragments of which are represented in Fig. 9.

The matrices of field emission cathodes possess the preset geometrical dimensions (diameter, step), they allow elimination of the screening effect and obtaining of homogeneous upon the surface and stable in time electron emission with the average current value of $40 \mu \mathrm{A}$ from a separate cathode at relatively low electric field intensity values. The cathode represented in Fig. 9(b) includes the cylinder container filled with a stock of the substance that decreases the output operation of the operating surface, which is represented by a continuous row of micro-elements forming up a "slot" L-cathode. Experimental results obtained while pilot testing of those cathodes at IRE NAS of Ukraine proved the opportunity for obtaining of such electron fluxes with high-value current density at not high values of accelerating voltages. 


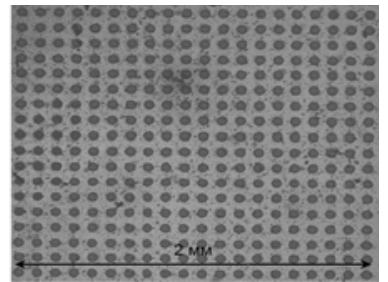

a)

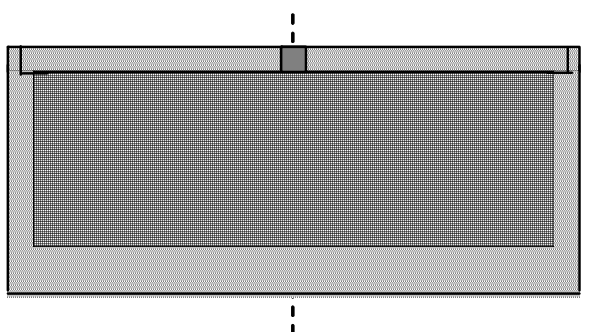

b)

FIG. 9: Microphotographic images of the field cathode surface [26] - a Block diagram of the "slot" L-cathode device [27] - b

\section{CONCLUSIONS}

The paper is considering the aspects of application of three types of focused beams of charged particles in the technology of manufacturing of nanodimensional components - electron beam lithography, focused beams of heavy ions and the PBW technology. It is demonstrated that as compared to the considered herein applications of focused electron beams and focused beams of low energy heavy ions, the PBW technology possesses higher potential capabilities at development of the small-dimension components for microoptics and nano-electronics.

\section{REFERENCES}

1. Ressier, L., Grisolia, J., Martin, C. et al., (2007), Ultramicroscopy, 107:985-988.

2. Lee, R.A. and Leech, P.W., (2007), Microelectronic Engineering, 84:669-672.

3. Gierak, J., Septier, A., and Vien, C., (1999), Nucl. Instr. and Meth. A 427:91-98.

4. Chyr, I. and Steck, A.J., (2001), J. Vac. Sci. Technol. B 19:2547-2550.

5. Watt, F., Bettiol, A.A., van Kan, J.A. et al., (2005), International Journal of Nanoscience. 4(3):269286.

6. Watt, F., Breese, M.B.H., Bettiol, A., and van Kan, J.A., (2007), Materials Today, 10(6):20-29.

7. Reyntjens, S. and Puers, R., (2001), J. Micromech. Microeng. 11:287-300.

8. Van Kan, J.A., Bettiol, A.A., Ansari, K. et al., (2004), Int. J. Nanotechnology, 1(4):464-479.

9. Melnik, K.I., Magilin, D.V., and Ponomarev, A.G., (2009), Nucl. Instr. and Meth. 267:2036-2040.

10. Mordyk, S.M., Miroshnichenko, V.I., Ponomarev, A.G. et al., (2004), Rev. Sci. Instr. 75(5):11401143.

11. Ignat'ev, I.G., Magilin, D.V., Miroshnichenko, V.I. et al., (2005), Nucl. Instr. and Meth. B. 231:94100.

12. Timoshenkov, S.P., Rubchits, V.G., Zotov, S.A., et al., (2007), Devices and Systems. Management, control, diagnostics. 2:60-63 (in Russian).

13. Kanyukov, Ye.Yu., Demyanov, S.Ye., and Petrov, A.V., (2008), 18-th International Crimean Conference "Microwave Equipment and Telecommunication Technologies" (CriMiCo'2008). Sevastopol, pp. 577-578 (in Russian). 
14. Ivanova, Yu.A., Ivanov, D.K., Fedotov, A.K. at al., (2007), Journal of Materials Science, 42(22):9163.

15. Mistry P., Gomez-Morilla, I., Grime, G.W. et al., (2005), Nucl. Instr. and Meth. B 237:188-193.

16. Ansari, K., van Kan, J.A., Bettiol, A.A., and Watt, F., (2006), J. Micromech. Microeng. 16:1967-1974.

17. Sum, T.C., Bettiol, A.A., van Kan, J.A. et al., (2003), Appl. Phys. Lett., 83:1707-1709.

18. Sum, T.C., Bettiol, A.A., Seng, H.L. et al., (2003), Nucl. Instr. and Meth. B. 210:266.

19. http://sofia.arc.nasa.gov

20. Finkel, M.I., Maslennikov, S.N., and Goltsman, G.N., (2005), Trans. Higher Education. Radio Physics. XLVIII(10-11):964-970 (in Russian).

21. Shestopalov, V.P. (ed.) (1991), Diffraction Oscillators, Naukova Dumka, Kiev:320 p. (in Russian).

22. Vorobyov, G.S., Petrovsky, M.V., and Krivets, A.S., (2006), Trans. Higher Education. Radio Electronics. 49(7):56-61 (in Russian).

23. Vorobyov, G.S., Petrovsky, M.V., Tsvyk, A.I., Khutoryan, E.M., and Tsvyk, L.I., (2005), Trans. Sumy National University. Series: Physics, mathematics, mechanics. 4(76):159-173 (in Russian).

24. Vorobyov, G.S., Petrovsky, M.V., Ruban, A.I., Zhurba, V.O., Belous, O.I., and Fisun, A.I., (2007), Telecommunications and Radio Engineering. 66(20):1839-1862.

25. Nanasheva, Ye.A., Trubitsina, O.N., Kartenko, N.F., and Usov, O.A., (1999), Solid State Physics. 41(5):882-884 (in Russian).

26. Solovey, D.V., Sakharuk, V.N., Novitsky, A.M. et al., (2009), 19-th International Crimean Conference "Microwave Equipment and Telecommunication Technologies” (CriMiCo'2009). Sevastopol, pp. 601-604 (in Russian).

27. Belousov, Ye.V., Zavertannyi, V.V., and Nesterenko, A.V., (2006), Radio Physics and Electronics. 11(2):275-280 (in Russian).

Volume 69, Number 4, 2010 